\title{
Entwicklung eines Konzepts zur Managementbewertung nach DIN EN ISO 9001
}

\author{
Patrick Liedtke $\cdot$ Hannes Feuersenger $\cdot$ Hans-Knud Arndt
}

Eingegangen: 12. Juli 2021 / Angenommen: 5. November 2021 / Online publiziert: 1. Dezember 2021

(C) Der/die Autor(en) 2021

Zusammenfassung Um wettbewerbsfähig zu bleiben, streben viele Organisationen eine kontinuierliche Verbesserung mithilfe eines Qualitätsmanagementsystems an. Die Zertifizierung spielt dabei zunehmend eine größere Rolle. Für den Bereich der Qualitätsmanagementsysteme besteht die Möglichkeit, sich nach der DIN EN ISO 9001 zertifizieren zu lassen. Um die Zertifizierung zu erreichen, muss eine sogenannte Managementbewertung durch die oberste Leitung vorgenommen werden, in der die relevanten Bereiche der Organisation evaluiert werden. Ziel ist es, Verbesserungspotenziale rechtzeitig zu erkennen und Korrekturmaßnahmen einzuleiten. Das Vorhaben birgt Schwierigkeiten, da es verschiedene Vorlagen und Definitionen gibt, welche unterschiedliche Inhalte und Ziele aufweisen. Dadurch ist oftmals unklar, welche Aspekte innerhalb der Managementbewertung beziehungsweise zu welchen Detaillierungsgrad diese abgebildet werden sollen. Aus diesem Grund stellt dieser Beitrag ein Konzept vor, um die Managementbewertung einheitlich und normgerecht abzubilden. Das Konzept soll Organisationen eine Hilfestellung für die Erstellung der Managementbewertung bieten. Dabei wurde für die Evaluation eine Befragung in einem mittelständischen Unternehmen durchgeführt.

Schlüsselwörter Managementbewertung · Qualitätsmanagementsysteme · DIN EN ISO $9001 \cdot$ Qualitätsmanagement · Qualität

Patrick Liedtke $(\bowtie) \cdot$ Hannes Feuersenger $\cdot$ Hans-Knud Arndt

Fakultät für Informatik, Arbeitsgruppe Wirtschaftsinformatik - Managementinformationssysteme, Otto-von-Guericke-Universität Magdeburg, Universitätsplatz 2, 39106 Magdeburg, Deutschland

E-Mail: patrick.liedtke@ ovgu.de

Hannes Feuersenger

E-Mail: hannes.feuersenger@ovgu.de

Hans-Knud Arndt

E-Mail: hans-knud.arndt@iti.cs.uni-magdeburg.de 


\title{
Development of a concept for management review according to DIN EN ISO 9001
}

\begin{abstract}
To stay competitive, many organizations strive for continuous improvement with the help of a quality management system. Certification is playing an increasingly important role in this process. In the area of quality management systems, it is possible to be certified in accordance with DIN EN ISO 9001. To achieve certification, a so-called management assessment must be carried out by the top management, in which the relevant areas of the organization are evaluated. The aim is to identify potential for improvement in good time and to initiate corrective measures. Such an endeavor is difficult because there are various templates and definitions, which have different contents and objectives. Thus, it is often unclear which aspects should be mapped within the management review or to which level of detail. For this reason, this paper presents a concept to map the management evaluation in a uniform and standard-compliant way. The concept is intended to provide organizations with assistance in the preparation of management reviews. For the evaluation, interviews were conducted in a medium-sized company.
\end{abstract}

Keywords Management review · Quality management systems · DIN EN ISO 9001 · Quality management · Quality

\section{Einleitung}

Egal ob bei Produkten, Dienstleistungen, Prozessen - Qualität, also der „Grad, in dem ein Satz inhärenter Merkmale eines Objekts (...) Anforderungen (...) erfüllt““ (DIN 2015a, Abschn. 3.6.2), ist einer der zentralen Leistungsparameter und sollte $\mathrm{zu}$ jeder Zeit berücksichtigt und gesteigert werden. Um die kontinuierliche Steigerung der Qualität zu gewährleisten, wurde das Konzept des Qualitätsmanagements eingeführt. Neben dem Festlegen der Qualitätspolitik und der -ziele gehören die Qualitätsplanung, -steuerung, -sicherung und -verbesserung zu den zentralen Aufgaben des Qualitätsmanagements (DIN 2015a, Abschn. 3.3.4).

Wie in der Praxis jedoch zu erkennen ist, wird die oberste Leitung einer Organisation oftmals unzureichend in die Geschehnisse des Qualitätsmanagements einbezogen (Harmeier 2017, S. 1). Aus diesem Grund fordert die DIN EN ISO 9001 (DIN 2015b, Abschn. 9.3) die verpflichtende Erstellung einer Managementbewertung. In der Praxis lässt sich schnell erkennen, dass die Erstellung einer solchen Bewertung eine Herausforderung darstellt: Während die Literatur auf der einen Seite von einem frei gestaltbaren Verfahren spricht, in dem die Geschäftsleitung die Themen einer Managementbewertung festlegt (Harmeier 2017, S. 8), existieren andererseits eine Vielzahl an Unsicherheiten über die zu betrachtenden Bewertungskriterien. Dem zugrunde liegt die ledigliche Nennung von Bewertungsaspekten ohne weitere Erläuterungen innerhalb der Norm. Die häufig unzureichende Einbindung der obersten Leitung zeigt sich auch daran, dass darauf hingewiesen wird, dass die Aufgabe nicht delegiert werden darf (Schulze 2021). Ein weiterer Grund für die 
existierenden Probleme in der Unternehmenspraxis ist das Fehlen eines einheitlichen Verfahrens und einer einheitlichen Vorlage für die Managementbewertung.

Aus diesem Grund beschreibt dieser Beitrag ein allgemeingültiges Konzept, welches alle Normanforderungen abbildet und die Geschäftsleitung bei der Managementbewertung unterstützen soll. Dafür werden zunächst bisherige Ansätze zur Unterstützung der Managementbewertung untersucht. Die Stärken der bisherigen Ansätze fließen daraufhin konsolidiert in das neu entwickelte Konzept ein, während die Schwächen eliminiert wurden. Anhand des soeben skizzierten Forschungsprozesses ergeben sich die folgenden Forschungsfragen, die im Rahmen dieses Beitrags geklärt werden:

FF1: Welche bisherigen Ansätze existieren zur Unterstützung der Managementbewertung und worin liegen dabei die Stärken und Schwächen?

FF2: Welche Anforderungen muss ein allgemeingültiges Konzept erfüllen, das Unternehmen zur eigenständigen und ganzheitlichen Durchführung einer Managementbewertung nach der DIN EN ISO 9001 befähigt?

Im nachfolgenden Kapitel erfolgt eine detaillierte Vorstellung des Forschungsprozesses. Das dritte Kapitel betrachtet den aktuellen Stand zur Managementbewertung und beinhaltet verschiedene Ansätze und Vorlagen im Vergleich, was die Forschungsfrage FF1 klärt. Nach der kritischen Bewertung des aktuellen Standes wird ein Konzept zur Managementbewertung im Kap. 4 vorgestellt, welches auf die Forschungsfrage FF2 eingeht. Eine Evaluation des Konzepts erfolgt im Kap. 5 anhand einer Fallstudie. Die wichtigsten Erkenntnisse und einen Ausblick auf weitere Forschungsarbeiten werden abschließend im sechsten Kapitel gegeben.

\section{Forschungsprozess}

Der verwendete Forschungsprozess orientiert sich an den Phasen Praxisbeobachtung und -analyse, Theoretische Arbeit, Empirische Arbeit und Ergebnisdarstellung. Dabei ergibt sich aus einem Problem, welches im Rahmen einer Analyse der Unternehmenspraxis identifiziert wurde, eine Forschungsfrage (Meyen et al. 2019. S. 48ff.). Um einen Bezug zur Forschungsfrage herzustellen, erfolgt eine Analyse und Beschreibung der Theorie (Mayring 2014, S. 10f.), wobei zusätzlich ein Forschungsdesign gewählt werden muss, welches zur Forschungsfrage passt (Ritschl et al. 2016, S. 10). Für die empirische Arbeit ist eine Datengrundlage notwendig, bestehend aus Dokumenten, Personenbefragungen und Situationen (Mayring 2014, S. 12). Die letzte Phase ist die Auswertung der Ergebnisse sowie eine Betrachtung dieser im Zusammenhang mit der Theorie und deren Weiterentwicklung (Ritschl et al. 2016, S. 10).

Die genannten Schritte werden in Abb. 1 auf die hier vorgestellte Forschung angewendet. Den Startpunkt stellt die im Rahmen der Praxisanalyse herausgekommene Beobachtung dar: Bei der Erstellung einer Managementbewertung treten in der Praxis häufig Unsicherheiten auf, da die Eingaben für die Managementbewer- 


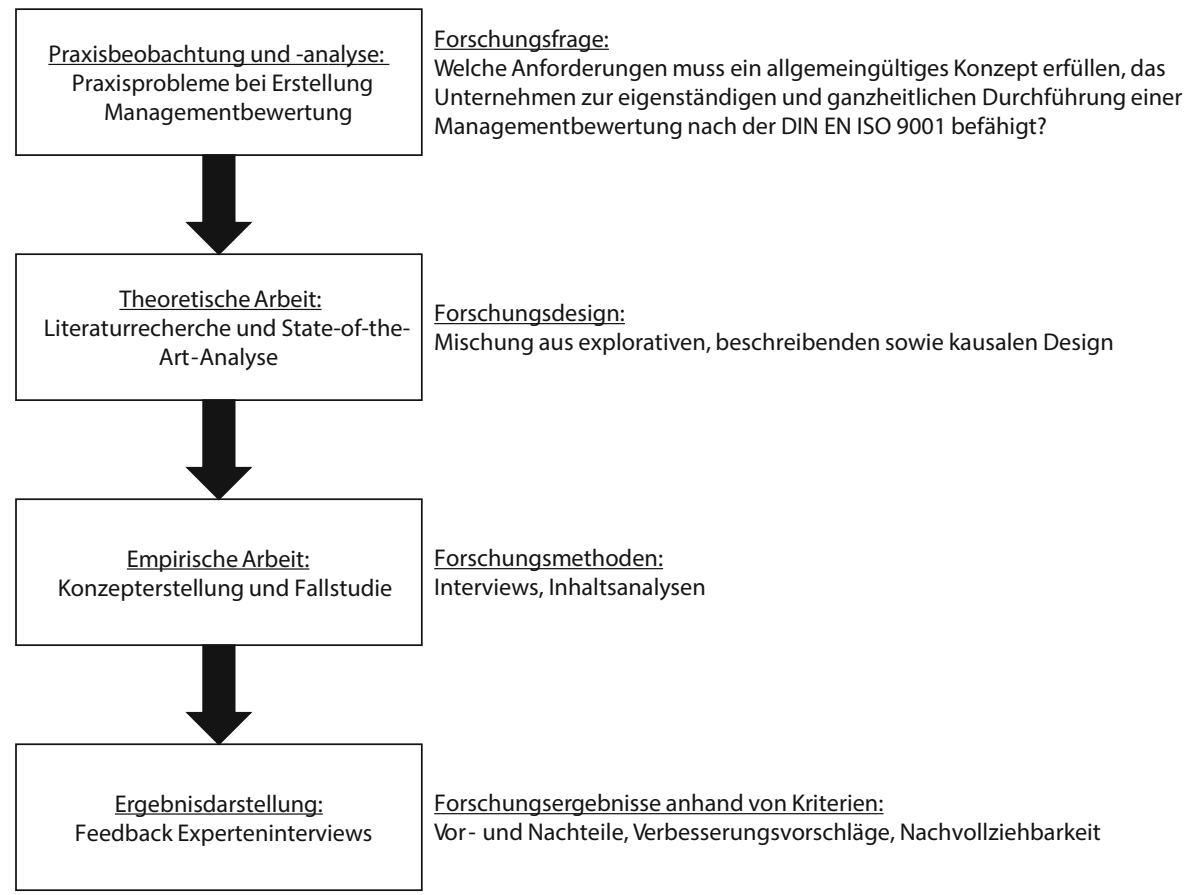

Abb. 1 Forschungsprozess

tung zwar festgelegt sind, aber weiterführende Informationen fehlen. Daraus ergibt sich die bereits beschriebene Forschungsfrage. Im nächsten Schritt folgt eine detaillierte Auseinandersetzung mit dem Forschungsgebiet durch eine Untersuchung von existierenden Vorlagen zur Managementbewertung. Diese Untersuchung bildet die Grundlage für die Entwicklung des Konzeptes für die Managementbewertung. Da neue Ansätze formuliert, bestehende Ansätze beschrieben werden sowie ein Konzept entwickelt wird, kann das Forschungsdesign gemäß der Definition von Mayring (2014) als Mischung von explorativen, beschreibenden sowie kausalen Design aufgefasst werden. Die Entwicklung des Konzepts sowie eine anknüpfende Fallstudie werden der Phase der empirischen Arbeit zugeordnet, wobei als Forschungsmethoden Interviews und Inhaltsanalysen zum Einsatz kommen. Die abschließende Ergebnisdarstellung stellt das Feedback aus den Experteninterviews dar, wobei dieses anhand verschiedener Kriterien bestimmt wird.

\section{Ansätze und Vorlagen der Managementbewertung}

Um die untersuchten Vorlagen zu evaluieren, werden Kriterien anhand von Fragestellungen definiert:

- Konformität zur Norm: Werden die Inhalte der Managementbewertung nach der DIN EN ISO 9001 in der Vorlage abgebildet? 
- Übersichtlichkeit: Besitzt die Vorlage einen logischen Aufbau?

- Nachvollziehbarkeit: Sind die Inhalte und mögliche Hilfestellungen verständlich?

- Nutzbarkeit: Ist eine Nutzung der Vorlage ohne zusätzliche Dokumente oder Hilfsmittel möglich?

Die folgende Bewertung und damit die Definition der Kriterien erfolgte nach gemeinsamer Einschätzung innerhalb eines Gremiums aus Experten der Praxis und der Forschung. Hierzu gehörte ein wissenschaftlicher Mitarbeitender aus dem Bereich Qualitätsmanagement sowie aus der Praxis ein Qualitätsmanagementbeauftragter, ein Projektleitender im Qualitätsmanagement sowie die Person, die für die Konzeptentwicklung verantwortlich ist. Ziel war es hierdurch, die Subjektivität der Entscheidung zu reduzieren, indem verschiedene Rollen und Perspektiven bei der Bewertung abgedeckt wurden. Das Kriterium zur Konformität der Norm wurde gewählt, weil nur hierdurch sichergestellt werden kann, dass das gesamte Managementsystem kontinuierlich bewertet wird. Die weiteren genannten Kriterien Übersichtlichkeit, Nachvollziehbarkeit und Nutzbarkeit versuchen die Belange der Anwender in die Evaluation einzubeziehen. Da der Anwender oftmals begrenzte zeitliche Ressourcen besitzt, um eine Managementbewertung anzufertigen, sollen die Inhalte der Vorlagen verständlich und vor allem übersichtlich dargestellt werden. Ebenfalls muss eine Vorlage bereits vollumfänglich nutzbar sein, da eine Anpassung sowie eine weitere Recherche zusätzlicher Dokumente und Hilfsmittel den Prozess verlängern würde. Anhand der genannten Kriterien kann eine differenzierte Beurteilung, sowohl aus der Anwendersicht als auch aus der Perspektive der Norm heraus, erfolgen. Die vorgenommene Evaluation soll dazu dienen, geeignete Eigenschaften für das neue Konzept zu identifizieren.

Bei der Auswahl der betrachteten Vorlagen wurden Vorlagen unterschiedlichster Organisationen eingebunden. So wurden Vorlagen von Zertifizierern, spezialisierten Unternehmen und branchenspezifische Vorlagen berücksichtigt und bewertet. Demgegenüber stehen Ausschlusskriterien, welche genutzt wurden, um zu entscheiden, dass Vorlagen nicht betrachtet werden:

- Automatisierte Vorlagen (Gefahr: das Dokument wird nicht nochmal angeschaut oder das Unternehmen muss eine neue Software dafür nutzen)

- Vorlagen, die lediglich ein Abbild der Norm darstellen

- Vorlagen, die nicht dem aktuellen Stand der DIN EN ISO 9001 von 2015 entsprechen

Insgesamt wurden fünf verschiedene Vorlagen betrachtet. Die Vorlage von Rohde Beratungen (Rohde 2016) ist sehr ausführlich gestaltet und deckt daher viele der Kriterien ab, sodass diese in der Praxis genutzt werden kann. Lediglich weitere Inhalte, die in der DIN EN ISO 9001 genannt werden, müssen normgerecht ergänzt werden. Durch eine Einleitung, eine Zusammenfassung und die Unterteilung der Vorlage ist diese sowohl übersichtlich als auch nachvollziehbar.

In dem vorgeschlagenen Protokoll der ORGAimprove GmbH (2016) werden eine vereinfachte Bewertung vorgenommen sowie die daraus resultierenden und einzuleitenden Maßnahmen aufgelistet, wobei eine Beschreibung separat vorgenommen werden kann. Die Bewertung erfolgt auf Grundlage eines Farbsystems, welches po- 
Tab. 1 Vergleich der Vorlagen nach den Kriterien

\begin{tabular}{llllll}
\hline Kriterium & $\begin{array}{l}\text { Rohde } \\
\text { Beratungen }\end{array}$ & $\begin{array}{l}\text { ORGAimprove } \\
\text { GmbH }\end{array}$ & $\begin{array}{l}\text { TÜV } \\
\text { Rheinland }\end{array}$ & $\begin{array}{l}\text { Selbst- } \\
\text { bewertung }\end{array}$ & $\begin{array}{l}\text { Ambulanter } \\
\text { Pflegebereich }\end{array}$ \\
\hline Konformität zur & - & - & + & + & - \\
Norm & & & & & + \\
Übersichtlichkeit & + & - & - & - & - \\
Nachvollziehbarkeit & + & - & + & + & - \\
Nutzbarkeit & + & - & - & - & - \\
\hline
\end{tabular}

sitiv hervorzuheben ist. Aufgrund der vereinfachten Darstellung existiert für alle genannten Kriterien jedoch ein gewisses Optimierungspotenzial.

Der $T \ddot{U} V$ Rheinland stellt eine Dokumentation mit vier Arbeitshilfen (Thomann 2021; Harmeier 2017) zur Verfügung und erfüllt damit die Maßgabe einer ausreichenden Dokumentation. Zu den Arbeitshilfen gehören eine Verfahrensanweisung, ein Managementbewertungsraster, ein Maßnahmenplan sowie ein Managementbewertungsprotokoll. Aufgrund der verschiedenen Dokumente entsteht ein erhöhter Verwaltungsaufwand, welcher die Übersichtlichkeit und die Nutzbarkeit einschränkt.

Seitens der Normung existiert als Arbeitshilfe die Selbstbewertung, verankert in der DIN EN ISO 9004 (DIN 2018). Um Stärken und Schwächen innerhalb der Organisation zu identifizieren, werden Selbstbewertungen genutzt (DIN 2018, Abschn. 10.6). Dabei erfolgt eine Evaluierung über die relevanten Themen anhand von Reifegraden, wobei diese Form nur als Vorbereitung für die Managementbewertung dient, welches die Nutzbarkeit als Vorlage dieser einschränkt. Die relevanten Themen bilden die Unterabschnitte der DIN EN ISO 9004 und können organisationsspezifisch angepasst werden (DIN 2018, Abschn. A.3).

Eine weitere Vorlage, die im ambulanten Pflegebereich genutzt werden kann (Schmidt 2010, S. 131), schlägt keine Gliederung der Managementbewertung vor, sondern listet Eingaben für diese auf. Zudem wird dabei erwähnt, dass bei dieser Bewertung die Balanced Scorecard als zusätzliches Bewertungsinstrument genutzt werden kann (Schmidt 2010, S. 131). Die Auflistung der Eingaben stellt lediglich einen Teil der in der Norm geforderten Themen dar, sodass die Kriterien der Konformität, der Nachvollziehbarkeit sowie der Nutzbarkeit nicht vollständig erfüllt werden.

Eine Gesamtbewertung der Vorlagen ist in der Tab. 1 ersichtlich. Da keine der Vorlagen gänzlich den Kriterien widerspricht, wurde eine Bewertung anhand von „,+“ und „,-“ vergeben. Dabei bedeutet ,+“" bei der Konformität der Norm, dass alle Normaspekte abgebildet werden und „,-“, dass Aspekte der Norm fehlen. Bei der Übersichtlichkeit steht „,+“ dafür, dass die Vorlage übersichtlich und logisch aufgebaut ist, und ,--“, dass es übersichtlich ist, aber trotzdem Verbesserungspotenzial existiert. Die Nachvollziehbarkeit wird mit „,+“ bewertet, wenn die Inhalte und Hilfestellungen zum Verständnis des Themas dienen, und mit ,--“, sofern die Inhalte verständlich sind, aber keine weiteren Hilfestellungen bieten. Das Kriterium der Nutzbarkeit wird dadurch unterschieden, ob eine Vorlage mit minimaler Anpassung einsetzbar ist $(,,+“)$ oder es einer größeren Anpassung bedarf beziehungsweise diese nur als Vorbereitung oder Übersicht dienen kann (,--“). In der Tabelle ist erkennbar, dass keine der untersuchten Vorlagen vollumfänglich die Kriterien erfüllen. Stär- 
ken und Schwächen konnten somit anhand der Bewertung identifiziert werden. Das bedeutet, dass eine vollumfängliche Lösung konzipiert werden muss, welche alle genannten Kriterien vereint.

\section{Konzept zur Managementbewertung}

Das entwickelte Konzept der Managementbewertung orientiert sich an den in der DIN EN ISO 9001 genannten Themen und ist dementsprechend aufgebaut. Die Limitierung des Konzepts auf die genannte Norm erfolgt, da die Managementbewertung innerhalb der genannten Norm gefordert wird. Für alle Teilbereiche des Konzeptes wurde ein Bewertungssystem basierend auf der Vorlage der ORGAimprove GmbH (2016) eingeführt. Dieses orientiert sich an den Farben einer Ampel und gibt der Organisation ein schnelles visuelles Feedback, ob Handlungsbedarf besteht. Dabei bedeutet Grün, dass alles im Plan ist und somit keine Maßnahmen notwendig sind. Eine Verfolgung sowie gegebenenfalls Maßnahmen sind bei Gelb notwendig, während bei Rot definitiv Maßnahmen veranlasst werden müssen. Die ergänzende Farbe Blau bedeutet, dass die Betrachtung nicht relevant ist und somit keine Maßnahmen notwendig sind.

Dieses Ampelsystem wird sowohl für die einzelnen Themen, aber auch in allen Unterthemen verwendet. Dabei kann die Farbe Blau nicht für die übergeordneten Themen aus der DIN EN ISO 9001 (DIN 2015b) verwendet werden, da diese in der Norm gefordert werden und somit für alle Organisationen relevant sind.

Bevor im folgenden Abschnitt eine Kurzfassung der Beschreibung des Konzeptes anhand der einzelnen Aspekte ${ }^{1}$ erfolgt, stellt die Abb. 2 einen Überblick über das Konzept der Managementbewertung dar, in dem alle Aspekte kurz beschrieben werden.

In der Norm DIN EN ISO 9001 wird als erster Aspekt Status von Maßnahmen vorheriger Managementbewertungen (1.) genannt, wobei eine Auflistung der Maßnahmen erfolgen soll. Diese Auflistung kann sich am Maßnahmenplan des $T \ddot{U} V$ Rheinlands (Thomann 2021) orientieren, wobei eine Unterteilung nach abgeschlossenen und offenen Maßnahmen vorgenommen werden kann. Eine Dokumentation über Besprechungen zu den Maßnahmen wird vorgeschlagen, um festzustellen, ob weitere Maßnahmen notwendig sind.

Ein weiteres Thema der Managementbewertung sind die Veränderungen bei internen und externen Themen, die das Qualitätsmanagementsystem betreffen (2.). Beispiele für diese Themen werden in der DIN EN ISO 9004 (DIN 2018, Abschn. 5.3) benannt, welche für dieses Konzept erweiterbar sind. Die Idee dabei ist, in der Managementbewertung pro Thema auszusagen, ob es Veränderungen gibt und ob es Einfluss auf die Bereiche nimmt. Daran lässt sich mittels des Ampelsystems festlegen, ob Maßnahmen für die jeweiligen Themen notwendig sind. Die Tab. 2 veranschaulicht eine mögliche Vorlage für die Abbildung von externen Themen.

\footnotetext{
${ }^{1}$ Für eine ausführlichere Beschreibung sowie Vorlagen für das Konzept der Managementbewertung siehe Onlinematerial 1.
} 


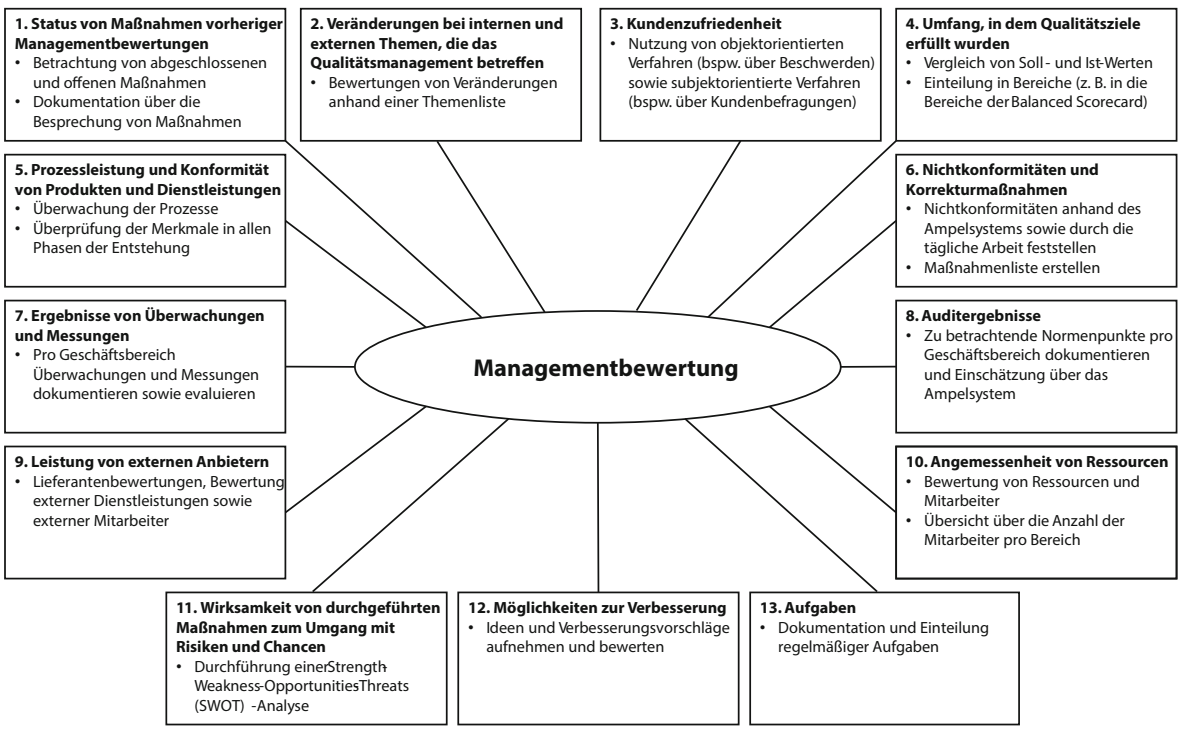

Abb. 2 Überblick über das Konzept der Managementbewertung

Tab. 2 Ausschnitt der Vorlage für Veränderungen bei externen Themen

\begin{tabular}{llll}
\hline Thema & $\begin{array}{l}\text { Veränderungen }- \\
\text { ja oder nein? }\end{array}$ & $\begin{array}{l}\text { Wenn ja - welche?/Einfluss } \\
\text { auf welche Bereiche? }\end{array}$ & $\begin{array}{l}\text { Maßnahmen } \\
\text { notwendig? }\end{array}$ \\
\hline $\begin{array}{l}\text { Gesetzliche und behördli- } \\
\text { che Anforderungen }\end{array}$ & $\ldots$ & $\ldots$ & 0 \\
$\ldots$ & $\ldots$ & $\ldots$ & $\ldots$ \\
\hline
\end{tabular}

Der nächste Aspekt, der in der Managementbewertung betrachtet wird, ist die Kundenzufriedenheit (3.). Die Kundenzufriedenheit lässt sich mithilfe objektorientierter (beispielsweise über Reklamationen) sowie subjektorientierter Verfahren (beispielsweise durch Befragung der Kunden) bestimmen (Zollandz et al. 2016, S. 606f.). Für das erste Verfahren können unter anderem die Anzahl der Beschwerdefälle beziehungsweise Reklamationen in Relation zur Anzahl der verkauften Produkte gesetzt werden. Für die subjektorientierte Verfahrensweise können klassische Fragebögen genutzt werden, in denen organisationsspezifische Abfragekriterien definiert wurden. Die Standardmetrik Customer Satisfaction Score gibt dabei die Durchschnittswerte der Kundenzufriedenheit an (Geer 2017). Die jeweils ermittelten Werte können mit den Werten aus den vorherigen Managementbewertungen verglichen werden, sodass über mögliche Maßnahmen mithilfe des Ampelsystems entschieden werden kann.

In dem Aspekt Umfang, in dem Qualitätsziele erfüllt wurden (4.), soll die Erreichung der Qualitätsziele mittels einer Soll-Ist-Analyse dokumentiert werden. Es empfiehlt sich, vorab Grenzwerte für eine Abweichung festzulegen, sodass eine Grundlage für die Beurteilung über mögliche Maßnahmen existiert. Die Ziele können verschiedenen Bereichen zugeordnet werden, die sich an der Methode der Balanced Scorecard, welche verschiedene Perspektiven betrachtet (Kaplan et al. 1997, S. 9), orientieren können. Daraus ergeben sich folgende Bereiche: Finanzbe- 
reich, Kundenbereich, Interner Prozessbereich und Lern- und Entwicklungsbereich. Im Rahmen der Managementbewertung soll ebenfalls der Aspekt der Prozessleistung sowie die Konformität von Produkten und Dienstleistungen (5.) betrachtet werden. Bei der Prozessleistung müssen folgende vier Indikatoren betrachtet werden (Förtsch und Meinholz 2018, S. 562): Kundenzufriedenheit, Kosten, Qualität und Zeit. Durch eine regelmäßige Überwachung der Prozesse soll die Prozessleistung bewertet und verbessert werden. Die Produktkonformität soll damit sichergestellt werden, dass die geforderten Merkmale während des gesamten Entstehungsprozesses geprüft werden (Rohde 2016, S. 15), wobei dies ebenfalls für Dienstleistungen geschehen soll. Aufbauend darauf ist der Aspekt Nichtkonformitäten und Korrekturmaßnahmen (6.) in der Norm genannt, welcher für eine genauere Betrachtung der mittels des Ampelsystems rot markierten Bereiche innerhalb der Managementbewertung genutzt werden kann. Die daraus resultierenden Maßnahmen können in einer Liste zusammengefasst werden, welche sich an dem Maßnahmenplan des TÜV Rheinland (Thomann 2021) orientiert und in die unterschiedlichen Geschäftsbereiche aufgeteilt werden kann.

Die Ergebnisse von Überwachungen und Messungen (7.) im Qualitätsmanagementsystem sind ebenfalls Bestandteil der Bewertung. Dabei kann beispielsweise eine Dokumentation der Überwachungen und Messungen gegliedert nach dem Geschäftsbereich und den zu betrachtenden Aspekten durchgeführt werden, wobei Anpassungen, wenn diese erforderlich sind, vorgenommen werden sollen. An der Stelle kann ebenfalls die Selbstbewertung aus der DIN EN ISO 9004 genutzt werden, wobei auf allen Ebenen der Organisation die Bewertung anhand von Reifegraden vorgenommen werden soll (DIN 2018, Tabelle A.27). Dabei können die Vorlagen aus der genannten Norm genutzt und die zu betrachtenden Unterabschnitte einem Verantwortlichen zugeordnet werden. In der Managementbewertung sollen ebenfalls die Auditergebnisse (8.) aufgeführt werden, wobei zu empfehlen ist, dass jeder Geschäftsbereich einzeln auditiert wird. Beim Audit soll überprüft werden, ob die Normvorgaben eingehalten werden. Dabei sind die Normpunkte sowie deren Unterpunkte aus der DIN EN ISO 9001 (DIN 2015b, Abschn. 5-10) zu beachten, wobei für jeden Geschäftsbereich vorab entschieden werden muss, ob die Normpunkte relevant sind. Dabei kann eine Matrix genutzt werden, in der in den Spalten die Geschäftsbereiche benannt sind und in den Zeilen die Norm(unter)punkte. Für die Protokollierung des Audits kann in der Matrix mittels des Ampelsystems angezeigt werden, an welchen Stellen Maßnahmen notwendig sind.

Unter dem Bestandteil Leistung von externen Anbietern (9.) muss die Qualität beziehungsweise das Qualitätsmanagement des Lieferanten bewertet werden. Die Bewertung ist dabei organisationsspezifisch und abhängig von der Art der Leistung. Es kann sich bei der Art der Leistung um Produkte, externe Dienstleistungen, aber auch um externe Mitarbeiter handeln, wobei unterschiedliche Kriterien zur Bewertung herangezogen werden sollen. Organisationen sollen bestrebt sein, die Beziehungen zu externen Anbietern zu pflegen und Partnerschaften aufzubauen (DIN 2018, Abschn. 9.6.). Demgegenüber steht eine Evaluierung der Angemessenheit von Ressourcen (10.). Eine Organisation soll ein Ressourcenmanagement nutzen, in dem ein Personal- und Beschaffungsmanagement abgebildet ist (Müller 2014, S. 84f.). Die bestellten Ressourcen können dann anhand verschiedener Kriterien bewertet werden. Da das Personal die ,wertvollste unternehmerische Ressource“ (Rohde 2016, 
S. 18) darstellt, soll die Entwicklung der Mitarbeiterzahlen tabellarisch nach Beschäftigungsform (Vollzeit, Teilzeit oder Studenten und Auszubildenden) aufgelistet werden, gegebenenfalls auch unterteilt in die Geschäftsbereiche.

Ein weiterer Aspekt der Managementbewertung ist eine Bewertung der Wirksamkeit von durchgeführten Maßnahmen im Hinblick auf Chancen und Risiken (11.). Diese Wirksamkeitsbewertung der Maßnahmen kann durch eine SWOT (StrengthWeakness-Opportunity-Threat)-Analyse erfolgen, wobei sowohl Stärken, Schwächen, Chancen als auch Risiken betrachtet werden (Bruhn 2020, S. 252f.). Um nach Abschluss der Maßnahme Beobachtungen und Messungen tätigen zu können, könnte die Wirksamkeitsbewertung ein Quartal oder ein Halbjahr nach dem Abschluss liegen. Für die Managementbewertung kann eine allgemeine SWOT-Analyse für jeden Geschäftsbereich vorgenommen werden. Während des Audits und auch allgemein treten Möglichkeiten zur Verbesserung (12.) und neue Ideen auf, die im Rahmen der Managementbewertung ebenfalls betrachtet werden. Bei der Besprechung der Ideen und Verbesserungsvorschläge sollen diese eingeschätzt und danach entschieden werden, ob eine Umsetzung erfolgt. Mit den genannten Punkten wären alle Aspekte einer Managementbewertung beantwortet, aber es empfiehlt sich weitere Aspekte einzubeziehen. Einer dieser Aspekte sind sich wiederholende Aufgaben (13.), die unterschiedliche Aspekte des Qualitätsmanagementsystems bearbeiten. Es ist sinnvoll Aufgabenlisten zu erstellen, wobei die Wiederholung der Aufgaben, der Stichtag, ein Verantwortlicher und ein Kontrolleur festgelegt werden soll.

\section{Evaluation des Konzepts}

Um das vorgestellte Konzept zu evaluieren, wurde eine Fallstudie in einem mittelständischen Unternehmen durchgeführt, welches in der IT-Dienstleistungsbranche tätig und nach der DIN EN ISO 9001 zertifiziert ist. Da Fallstudien unterschiedliche Quellen an Informationen nutzen (Schögel und Tomczak 2009, S. 82), werden in der beschriebenen sowohl die Managementbewertungen der vergangenen Jahre betrachtet als auch qualitative Interviews ausgewertet. Dabei wurden zunächst die Managementbewertungen aus den Jahren 2018 und 2019 analysiert. Hierbei konnte festgestellt werden, dass einige der zu betrachtenden Aspekte nicht ausführlich genug sind oder nicht berücksichtigt wurden. Die in der Praxis existierende Uneinigkeit zwischen den definierten Aspekten der Norm und der Aussage, dass die Geschäftsleitung die Themen der Managementbewertung bereitstellen soll (Harmeier 2017, S. 8), konnte somit auch in der Fallstudie identifiziert werden. Durch eine eigene Webanwendung im Unternehmen können einzelne Bereiche der Managementbewertung abgebildet und somit das Verfahren erleichtert werden. Im Unternehmen werden die Kennzahlen und Werte für die Managementbewertung vorbereitet, die dann während einer Besprechung zwischen der obersten Leitung und den Qualitätsmanagementbeauftragten diskutiert werden.

Im Rahmen der Fallstudie wurden qualitative Interviews geführt, mit dem Ziel Vor- und Nachteile, Verbesserungsvorschläge und weitere Ideen zu ermitteln sowie die Verständlichkeit und Nachvollziehbarkeit des neu entwickelten Artefakts zu evaluieren. Daher werden allgemein und pro Aspekt diese Informationen ab- 
gefragt. Als Erhebungsinstrument wurde ein semistrukturierter Interviewleitfaden erstellt. Dies bietet die Möglichkeit, dass die Interviewten weitere Anmerkungen zur Verbesserung des Konzepts einbringen. Da die Interviewpartner unter anderem aus unterschiedlichen Hierarchieebenen sein sollen (Eisenhardt und Graebner 2007, S. 28), wurden die Qualitätsmanagementbeauftragte sowie die Projektleiterin des Qualitätsmanagements befragt, wobei eine detaillierte Diskussion aller einzelnen Teilbereiche erfolgte. Als Gegenstand der Befragung diente das erstellte Konzept, welches die interviewten Personen vorab gelesen haben, um so die Fragen im Interview beantworten zu können.

Insgesamt wurde das Konzept als sehr verständlich und nachvollziehbar eingeschätzt, wobei die Anmerkungen dazu beitragen, das Konzept qualitativ zu verbessern. Dabei wurden unter anderem als Vorteile das Ampelsystem, festgelegte Verantwortlichkeiten sowie klare Kategorien, Themen und Skalen genannt. Auf der anderen Seite lässt sich erkennen, dass mithilfe des Konzepts der Verwaltungsaufwand an einigen Stellen trotzdem weiterhin existiert, allerdings kann dieser weiter minimiert werden. Als Beispiel wurde beim Status von Maßnahmen vorheriger Managementbewertungen ausgesagt, dass Gespräche mit dem Kontrolleur einer Maßnahme zeitintensiv und gegebenenfalls subjektiv sind. Des Weiteren wurde angemerkt, dass die Ansprechpartner sowie weitere Mitarbeiter zu bestimmten Themen bei der Vorbereitung einbezogen werden sollen, da ,das Management bei manchen Themen kein Experte für die Bewertung von Änderungen“"2 ist. Zudem ist das Problem der Bewertung der Kundenzufriedenheit erkennbar, da der Kunde oftmals nach seiner Gefühlslage beurteilt. Die Organisation muss aufbauend auf dem vorgeschlagenen Konzept eine Strategie für die Kundenzufriedenheit entwickeln. Das Konzept könnte weitere Vorschläge zur Verfügung stellen, wie zum Beispiel Zielvorschläge, allerdings muss die Organisation selbst einige Entscheidungen treffen, die die Umsetzung des Konzepts umfassen. Durch Zielvorschläge soll sichergestellt werden, dass nur messbare und vergleichbare Ziele gesetzt werden, wobei ansonsten die Gefahr besteht, dass dies nicht gemacht wird. Außerdem wurde in den Interviews angemerkt, dass externe Nichtkonformitäten oftmals größere Probleme als interne Nichtkonformitäten darstellen, sodass eine separate Betrachtung dieser erfolgen soll. Es wurde als Nachteil genannt, dass im betrachteten Konzept alle Nichtkonformitäten gleich behandelt werden, obwohl bei externen Nichtkonformitäten gegebenenfalls ein größerer Umsatzverlust entsteht ${ }^{3}$. Bei den Auditergebnissen kann es zum Fehlen einer langen Beschreibung kommen, da lediglich die Matrix abgebildet wird. Dem kann entgegengewirkt werden, indem eine ,Fußnote als Erläuterung bei Nichtkonformitäten" ${ }^{4}$ dient. Bei der Wirksamkeitsbewertung mithilfe der SWOT-Analyse wurde durch die befragten Personen angemerkt, dass es dabei zu längeren Diskussionen kommen kann, da die Definitionen von Stärken, Schwächen,

\footnotetext{
2 Ausschnitt aus einem Interview vom 04.09.2020 mit der Qualitätsmanagementbeauftragten im betrachteten Unternehmen.

3 Sinngemäße Wiedergabe aus dem Interview vom 09.09.2020 mit der Projektleiterin des Qualitätsmanagements im betrachteten Unternehmen.

4 Ausschnitt aus einem Interview vom 09.09.2020 mit der Projektleiterin des Qualitätsmanagements im betrachteten Unternehmen.
} 
Chancen und Risiken anders ausgelegt werden können. Als Verbesserungspotential können die Qualitätsmanagementbeauftragten die Bewertung der einzelnen Maßnahmen vornehmen ${ }^{5}$, während das Management die Bewertung der einzelnen Bereiche vornimmt. Die Verbesserungsvorschläge seitens der Mitarbeiter können durch eine Umfrage eingebracht werden. Die Anmerkungen zeigen Verbesserungspotential für das erstellte Konzept, allerdings ist eine eigenständige und ganzheitliche Durchführung der Managementbewertung innerhalb der Organisationen durch die im Konzept definierten Hilfestellungen gegeben.

Um Bezug auf die definierten Kriterien bei den bereits vorhandenen Vorlagen zu nehmen, lässt sich für das neue Konzept die Konformität zur Norm darin bestätigen, dass alle Aspekte, die in der Norm unter der Managementbewertung genannt werden, integriert wurden. Das Kriterium zur Übersichtlichkeit ist organisationsspezifisch zu bewerten, da es sich hierbei um ein Konzept handelt, welches an die Bedürfnisse der Organisation angepasst werden soll. Denkbar ist eine Nutzung der angefügten Vorlagen oder eine Nachbildung der theoretischen Aspekte in einer technischen Lösung. Die Vorlagen wurden in den Interviews als übersichtlich eingeschätzt. Die interviewten Personen wurden ebenfalls zur Nachvollziehbarkeit zu jedem Aspekt befragt. Dabei wurde ausgesagt, dass die Aspekte und die Vorlagen nachvollziehbar sind. Zur Nutzbarkeit wurde angemerkt, dass das Konzept innerhalb des Unternehmens genutzt werden kann.

\section{Zusammenfassung und Ausblick}

Im Rahmen der Arbeit wurde ein Konzept für die Managementbewertung entwickelt, welches Organisationen dabei unterstützt, die Inhalte dieser Bewertung vollständig abzubilden. Die Untersuchung des aktuellen Stands der Managementbewertung zeigt, dass es sich dabei um ein komplexes Verfahren handelt. Der Anwender wird mit der Definition aus der DIN EN ISO 9001 allein gelassen, da zu den einzelnen Inhalten keine Erläuterungen gegeben werden. Die betrachteten Vorlagen besitzen einige gute Ansätze, welche im beschriebenen Konzept Anwendung finden. Allerdings fehlen teilweise wichtige Inhalte beziehungsweise die Vorlagen können nicht genutzt werden, da sie branchenspezifisch sind.

Zur Evaluierung des Konzepts wurde eine Fallstudie in einem Unternehmen durchgeführt. Dabei konnte festgestellt werden, dass das entwickelte Konzept in der Praxis eingesetzt werden kann. Innerhalb der Fallstudie wurden Anmerkungen aufgenommen, die den ersten Entwurf des Konzepts verbessern. Einige Aspekte im Konzept lassen sich nicht allgemeingültig abbilden, sodass es an den Stellen einer organisationsspezifischen Anpassung bedarf. Grundsätzlich soll das Konzept dazu dienen, dass der Verwaltungsaufwand bei der Erstellung einer Managementbewertung verringert wird. Dazu ist eine technische Umsetzung des Konzepts empfehlenswert. In der weiteren Forschung zu diesem Thema müssen weitere Erkenntnisse durch Befragungen gesammelt werden, um das Konzept qualitativ zu verbessern. Ebenfalls

\footnotetext{
5 Sinngemäße Wiedergabe aus dem Interview vom 04.09.2020 mit der Qualitätsmanagementbeauftragten im betrachteten Unternehmen.
} 
muss der produktive Einsatz des beschriebenen Konzepts in den Organisationen beobachtet werden, sodass eine detailliertere Evaluierung vorgenommen werden kann. Des Weiteren besteht hierbei zunächst eine Einschränkung des Konzepts auf die DIN EN ISO 9001, aber in zukünftigen Arbeiten lässt sich außerdem evaluieren, ob das Konzept auch auf andere Qualitätsmanagement-Modelle angewendet werden kann, wie beispielsweise EFQM.

Zusatzmaterial online Zusätzliche Informationen sind in der Online-Version dieses Artikels (https://doi. org/10.1365/s40702-021-00809-w) enthalten.

Funding Open Access funding enabled and organized by Projekt DEAL.

Open Access Dieser Artikel wird unter der Creative Commons Namensnennung 4.0 International Lizenz veröffentlicht, welche die Nutzung, Vervielfältigung, Bearbeitung, Verbreitung und Wiedergabe in jeglichem Medium und Format erlaubt, sofern Sie den/die ursprünglichen Autor(en) und die Quelle ordnungsgemäß nennen, einen Link zur Creative Commons Lizenz beifügen und angeben, ob Änderungen vorgenommen wurden.

Die in diesem Artikel enthaltenen Bilder und sonstiges Drittmaterial unterliegen ebenfalls der genannten Creative Commons Lizenz, sofern sich aus der Abbildungslegende nichts anderes ergibt. Sofern das betreffende Material nicht unter der genannten Creative Commons Lizenz steht und die betreffende Handlung nicht nach gesetzlichen Vorschriften erlaubt ist, ist für die oben aufgeführten Weiterverwendungen des Materials die Einwilligung des jeweiligen Rechteinhabers einzuholen.

Weitere Details zur Lizenz entnehmen Sie bitte der Lizenzinformation auf http://creativecommons.org/ licenses/by/4.0/deed.de.

\section{Literatur}

Bruhn M (2020) Qualitätsmanagement für Dienstleistungen. Handbuch für ein erfolgreiches Qualitätsmanagement. Grundlagen - Konzepte - Methoden, 11. Aufl. Springer Gabler, Heidelberg https://doi. org/10.1007/978-3-662-62120-2

DIN Deutsches Institut für Normung e.V. (2015a) Qualitätsmanagementsysteme - Grundlagen und Begriffe (ISO 9000:2015), DIN EN ISO 9000:2015-11, Beuth Verlag, Berlin

DIN Deutsches Institut für Normung e.V. (2015b) Qualitätsmanagementsysteme - Anforderungen (ISO 9001:2015), DIN EN ISO 9001:2015-11, Beuth Verlag, Berlin

DIN Deutsches Institut für Normung e.V. (2018) Qualitätsmanagement - Qualität einer Organisation Anleitung zum Erreichen nachhaltigen Erfolgs (ISO 9004:2018), DIN EN ISO 9004:2018-08, Beuth Verlag, Berlin

Eisenhardt KM, Graebner ME (2007) Theory building from cases: opportunities and challenges. The Academy of Management Journal 50(1):25-32. https://doi.org/10.5465/amj.2007.24160888

Förtsch G, Meinholz H (2018) Handbuch betriebliches Umweltmanagement, 3. Aufl. Springer Vieweg, Wiesbaden https://doi.org/10.1007/978-3-658-19151-1

Geer C (2017) Kundenzufriedenheit messen: 5 sinnvolle Kennzahlen. https://www.honestly.de/blog/ kundenzufriedenheit-kennzahlen/. Zugegriffen: 14. Mai 2021

Harmeier J (2017) Managementbewertung nach ISO 9001:2015. TÜV Media, Köln

Kaplan RS, Norton DP, Horváth P (1997) Balanced scorecard. Strategien erfolgreich umsetzen. SchäfferPoeschel, Stuttgart

Mayring P (2014) Qualitative content analysis: theoretical foundation, basic procedures and software solution. Klagenfurt

Meyen M, Löblich M, Pfaff-Rüdiger S, Riesmeyer C (2019) Qualitative Forschung in der Kommunikationswissenschaft. Eine praxisorientierte Einführung, 2. Aufl. Springer VS, Wiesbaden https://doi.org/ 10.1007/978-3-658-23530-7

Müller E (2014) Qualitätsmanagement für Unternehmer und Führungskräfte. Was Entscheider wissen müssen. Springer Gabler, Wiesbaden https://doi.org/10.1007/978-3-642-41002-4 
ORGAiprove GmbH (2016) Excel-Vorlage Managementbewertung. http://www.orgaimprove.com/ infocenter/download/excel-tool-managementbewertung/. Zugegriffen: 14. Mai 2021

Ritschl V, Weigl R, Stamm TA (2016) Wissenschaftliches Arbeiten und Schreiben. Verstehen, Anwenden, Nutzen für die Praxis. Springer, Berlin Heidelberg https://doi.org/10.1007/978-3-662-49908-5

Rohde HU (2016) Vorlage Qualitätsbericht - Managementbewertung. https://rohde-qm-consult.de/wpcontent/uploads/2016/10/Review-Vorlage.pdf. Zugegriffen: 29. Apr. 2020

Schmidt S (2010) Das QM-Handbuch. Qualitätsmanagement für die ambulante Pflege, 2. Aufl. Springer, Heidelberg https://doi.org/10.1007/978-3-642-12646-8

Schögel M, Tomczak T (2009) Fallstudie. In: Baumgarth C, Eisend M, Evanschitzky H (Hrsg) Empirische Mastertechniken. Eine anwendungsorientierte Einführung für die Marketing- und Managementforschung. Springer Gabler, Wiesbaden, S 79-105 https://doi.org/10.1007/978-3-8349-8278-0_3

Schulze A (2021) Managementbewertung: 5 Gründe, weshalb Chefs diese lieben sollten. https://www. johner-institut.de/blog/qualitaetsmanagement-iso-13485/managementbewertung/. Zugegriffen: 30. Sept. 2021

Thomann H (2021) QM in Dienstleistungsunternehmen. 06100 Managementbewertung nach ISO 9001:2015. https://qmd.tuev-media.de/xhtml/document.jsf?docId=docs/qmd_0000006100.html\& alias=06100_EndAli\&anchor=\&event=navigation\#06100_04_doc_arb. Zugegriffen: 14. Mai 2021

Zollandz HD, Ketting M, Pfundtner R (2016) Lexikon Qualitätsmanagement. Handbuch des modernen Managements auf Basis des Qualitätsmanagements, 2. Aufl. De Gruyter, Berlin https://doi.org/10. 1515/9783486845204 